\title{
Doxycycline reverses epithelial-to-mesenchymal transition and suppresses the proliferation and metastasis of lung cancer cells
}

\author{
Yuan Qin ${ }^{1,2, *}$, Qiang Zhang ${ }^{1,2, *}$, Shan Lee ${ }^{1,2, *}$, Wei-long Zhong ${ }^{1,2, *}$, Yan-rong Liu ${ }^{2}$, \\ Hui-juan Liu' ${ }^{2}$ Dong Zhao ${ }^{1,2}$, Shuang Chen ${ }^{2}$, Ting Xiao ${ }^{1,2}$, Jing Meng ${ }^{1,2}$, \\ Xue-shuang Jing ${ }^{2}$, Jing Wang ${ }^{2}$, Bo Sun², Ting-ting Dai ${ }^{2}$, Cheng Yang ${ }^{1,2}$, Tao Sun ${ }^{1,2}$, \\ Hong-gang Zhou ${ }^{1,2}$ \\ ${ }^{1}$ State Key Laboratory of Medicinal Chemical Biology and College of Pharmacy, Nankai University, Tianjin, China \\ ${ }^{2}$ Tianjin Key Laboratory of Molecular Drug Research, Tianjin International Joint Academy of Biomedicine, Tianjin, China \\ *These authors have contributed equally to this work
}

Correspondence to:

Tao Sun, e-mail: sunrockmia@hotmail.com

Hong-gang Zhou, e-mail: honggang.zhou@vip.126.com

Keywords: doxycycline, antitumor, EMT, metastasis, lung cancer

Received: July 11,2015 Accepted: September 24, $2015 \quad$ Published: October 14, 2015

\section{ABSTRACT}

The gelatinase inhibitor doxycycline is the prototypical antitumor antibiotic. We investigated the effects of doxycycline on the migration, invasion, and metastasis of human lung cancer cell lines and in a mouse model. We also measured the effect of doxycycline on the transcription of epithelial-mesenchymal transition (EMT) markers, and used immunohistochemistry to determine whether EMT reversal was associated with doxycycline inhibition. Doxycycline dose-dependently inhibited proliferation, migration, and invasion of NCI-H446 human small cell lung cancer cells. It also suppressed tumor growth from NCI-H446 and A549 lung cancer cell xenografts without altering body weight, inhibited Lewis lung carcinoma cell migration, and prolonged survival. The activities of the transcription factors Twist1/2, SNAI1/2, AP1, NF-kB, and Stat3 were suppressed by doxycycline, which reversed EMT and inhibited signal transduction, thereby suppressing tumor growth and metastasis. Our data demonstrate functional targeting of transcription factors by doxycycline to reverse EMT and suppress tumor proliferation and metastasis. Thus, doxycycline selectively targets malignant tumors and reduces its metastatic potential with less cytotoxicity in lung cancer patients.

\section{INTRODUCTION}

Antibiotics that target mitochondria have great potential as anticancer drugs. [1]. For example, tetracyclines are cytotoxic to some tumor cells [2] and have been shown to induce apoptosis in osteosarcoma, prostatic cancer cells, and lymphocytes [4, 5]. Doxycycline, a semi-synthetic tetracycline, inhibits matrix metalloproteinase (MMP) activation and cell proliferation [3] and can also interfere with tumor-related protein synthesis in mammalian cells [6-8]. The selective permeability of different mammalian cells to doxycycline led to the hypothesis that it could be used to arrest cell proliferation and treat malignancies. Thus, doxycycline has been used in combination with targeted drugs in clinical trials with patients with advanced cancer.
Lung cancer is the most common cause of cancer mortality worldwide [9]. Tumor recurrence and metastasis are common in lung cancer despite various lines of standard therapy and the introduction of targeted agents. The vast majority of patients with lung cancer fail to respond to tyrosine-kinase inhibitors against the epidermal growth factor receptor (EGFR) [10], and no effective drug is available for small cell lung cancer $[11,12]$. Therefore, the development of alternative treatments is urgently needed to improve outcome.

To investigate the therapeutic efficacy and the mechanism of action of doxycycline in lung cancer, we investigated the effects of doxycycline on migration, invasion, and metastasis of various lung cancer cell lines. We also examined its functional interference in EMT. In 
addition, we used a tumor-bearing mouse xenograft model to investigate doxycycline inhibition of tumor growth in vivo.

\section{RESULTS}

\section{Doxycycline reduces cell viability and alters cell cycle dynamics in human lung cancer cell lines}

Using MTT assay, we determined the effect of $48 \mathrm{~h}$ treatment with doxycycline on cell viability of various cancer cell lines. As shown in Fig. 1A, lung cancer cells were more sensitive to doxycycline than most of the other cell lines. NCI-H446 and A549 cells showed sensitivity to doxycycline with $\mathrm{IC}_{50}$ values of $1.7043 \pm 0.1241$ and $1.0638 \pm 0.1266 \mu \mathrm{M}$, respectively. Proliferation of NCI-H446 (Fig. 1B) and A549 cells (Fig. 1C) was inhibited by doxycycline in a dose-dependent manner.

We also investigated whether doxycycline interferes with the cell cycle. NCI-H446 and A549 cells were treated with different doses of doxycycline for $24 \mathrm{~h}$, followed by cell cycle analysis using flow cytometry. Cells treated with doxycycline started to arrest at G0/G1 phase after treatment for $24 \mathrm{~h}$ (Fig. 1D \& 1E). After treatment, NCI-H446 cells at G0/G1 accounted for approximately $24 \%$ of the total cell population, and A549 cells in G0/G1 population accounted for approximately $44 \%$.

\section{Doxycycline inhibits lung cancer cell invasion and migration in vitro}

To study whether doxycycline inhibits NCI-H446 and A549 cell invasion we used Matrigel-coated transwell chambers. For both cell lines, when compared with the control group, the number of cell invasions through the Matrigel-coated filter was dose-dependently reduced by doxycycline (Fig. 2A \& 2B). Notably, the inhibitory effects of doxycycline on cell invasion were due to its cytotoxic effects, given that cell viability was also decreased at the concentration ranges tested.

Next we assessed the ability of doxycycline to inhibit the migration of NCI-H446 and A549 cells using a wound-healing assay. Confluent cells were scraped with a sterile pipette tip, and the remaining cells were allowed to migrate into the gap created in the absence or presence of doxycycline. Remarkably, after 24 and $48 \mathrm{~h}$ treatment, the wound gap of both cell types was wider in the doxycycline-treated groups than in the untreated groups (Fig. 2C \& 2D), indicating that doxycycline inhibits motility of both NCI-H446 and A549 cells. The cell growth curves of NCI-H446 and A549 were shown in Fig. S1

The degradation of the extracellular matrix (ECM) and basement membrane are crucial steps in cancer invasion and metastasis and the proteolytic enzymes MMP-2 and MMP-9 are involved in this process. We next measured the secretion of MMP-2 and MMP-9 from
NCI-H446 and A549 cells with or without doxycycline treatment. As shown in Fig. 2E, doxycycline inhibited MMP-2 and MMP-9 secretion into the medium in a dosedependent manner. This finding suggests that doxycycline may reduce lung cancer metastasis by inhibiting the degradation of the ECM and basement membrane.

\section{Doxycycline inhibits the expression of epithelial markers and changes cellular morphology}

Vimentin and E-cadherin regulate the expression of proteins involved in ECM degradation. Thus, we used immunofluorescent staining to measure the effect of doxycycline on vimentin and E-cadherin levels. NCI-H446 and A549 cells were treated with different doses of doxycycline for $24 \mathrm{~h}$,. In response to doxycycline treatment, vimentin expression decreased, whereas E-cadherin expression increased in both cell lines (Fig. 3A $\& 3 \mathrm{~B})$. We also tested the effect of doxycycline on the cellular morphology of NCI-H446 and A549 by HCS. In response to doxycycline treatment, the perimeter-toarea ratio decreased, whereas pyknosis increased in both NCI-H446 and A549 cells (Fig. 3C \& 3F). The relative area of nucleus also increased, whereas the DNA content in cells decreased in both NCI-H446 and A549 cells (Fig. 3D \& 3E). As shown in Fig. 3, the results of DNA reduction were comparisons of all cells, not only $\mathrm{S}$ stage cells. For A549 cells, as shown in Fig. 1E, the number of $\mathrm{S}$ stage cells did not differ between groups.

\section{Doxycycline inhibits the activity of EMT transcription factors}

NCI-H446 and A549 cells transfected with luciferase reporter plasmids for various gene promoters or response elements were treated with doxycycline for $48 \mathrm{~h}$. In NCI-H446 cells, doxycycline suppressed the expression of Twist1, Twist2, SNAI1, STAT-3, and NF-kB and promoted the expression of AP-1. However, no change in SNAI2 expression was observed (Fig. 4A). In A549 cells, doxycycline suppressed the expression of Twist1, Twist2, SNAI1, SNAI2, STAT-3, and NF- $\mathrm{KB}$ and promoted the expression of AP-1 (Fig. 4B). Multidimensional liquid chromatography-tandem mass spectrometry was performed to evaluate differentially expressed proteins in NCI-H446 cells after doxycycline treatment. 36 proteins were upregulated by doxycycline and 42 were downregulated. After doxycycline treatment in cancer cell lines, RPLs were directly or indirectly regulated. Some RPLs were inhibited by doxycycline, such as RPL23A, RPL10A, RPL12, RPL38 and RPL13. On the contrary, RPL3 was compensatorily increased to maintain basic protein synthesis. (Fig. 4C-4G). These proteins are involved in cell migration, cytoskeletal maintenance, cell proliferation, adhesion, and differentiation (Fig. 4H). 

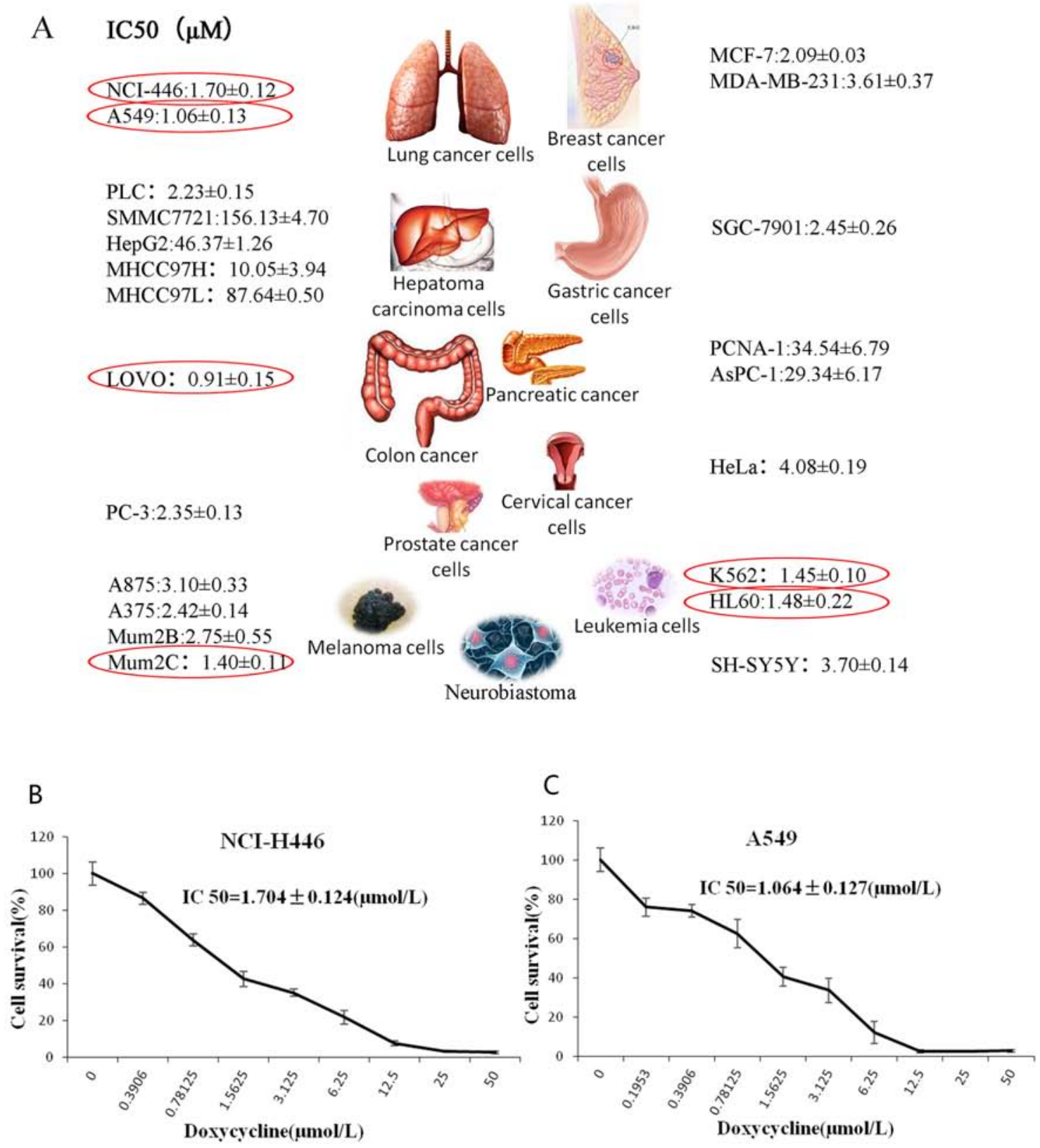

D

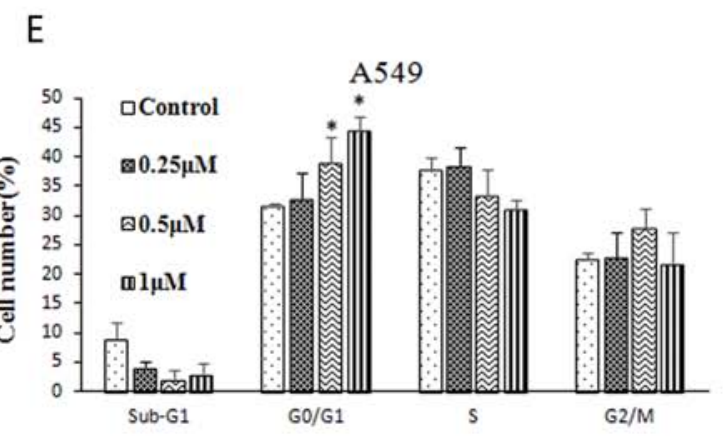

Figure 1: Effect of doxycycline on the cell viability and cell cycle. $\mathbf{A}$. $\mathrm{IC}_{50}(\mu \mathrm{M})$ dose of doxycycline for different cancer cell lines. Lung cancer cells were more sensitive to doxycycline than all the other cell lines $(P<0.05)$. B. Cell survival of NCI-H446 cells and C. A549 cells treated with the indicated amounts of doxycycline for $48 \mathrm{~h}$; IC50 $=1.7043 \pm 0.1241 \mu \mathrm{M}$ and $1.0638 \pm 0.1203 \mu \mathrm{M}$, respectively. D. NCI-H446 and E. A549 cells treated with different doses of doxycycline for $24 \mathrm{~h}$ were evaluated by fluorescence-activated cell sorting (FACS) analysis. Doxycycline induced cell cycle arrest at the G0/G1 phase in both cell lines $(P<0.05)$. Each experiment was performed in triplicate. Results show the means of the three experiments, and the error bars represent standard deviation $\left({ }^{*} P<0.05\right)$. 
A
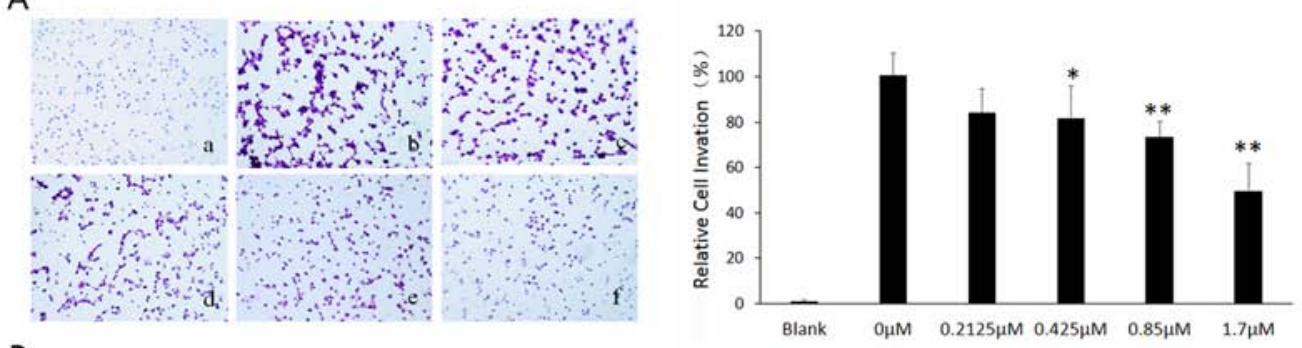

B
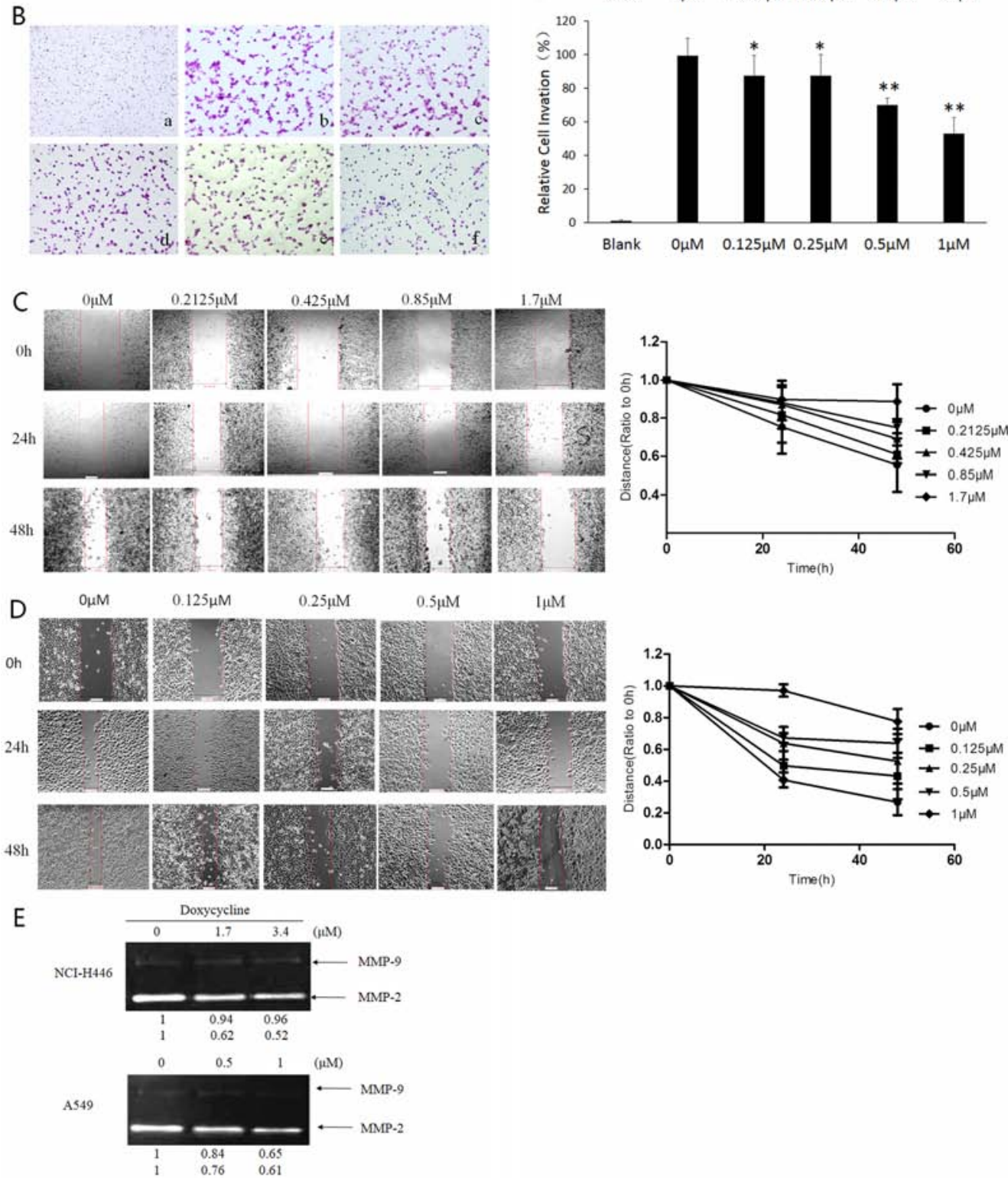

Figure 2: Effect of doxycycline on invasion, migration, and activities of matrix metalloproteinases (MMPs) of NCI-H446 and A549 cells. Transwell chambers were used for the invasion assay, and images were taken at $200 \times$ magnification. A. NCI-H446 cells were treated with 0 (b), 0.2125 (c), 0.425 (d), 0.85 (e), and $1.7 \mu \mathrm{M}$ (f) doxycycline for 24 h. No cells were seeded in (a). Doxycycline inhibited invasion of NCI-H446 cells $(P<0.05)$. B. A549 cells were treated with 0 (b), 0.125 (c), 0.25 (d), 0.5 (e), and $1 \mu$ M (f) of doxycycline for $24 \mathrm{~h}$. No cells were seeded in (a). Doxycycline inhibited invasion of A549 cells $(P<0.05)$. C. NCI-H446 cells were incubated in fetal bovine serum (FBS)-free medium containing $0,0.2125,0.425,0.85$, or $1.7 \mu \mathrm{M}$ doxycycline for 24 or $48 \mathrm{~h}$. Doxycycline inhibited the migration of NCI-H446 cells $(P<0.05)$. D. A549 cells were incubated in FBS-free medium containing $0,0.125,0.25,0.5$, or $1 \mu \mathrm{M}$ doxycycline for 24 or $48 \mathrm{~h}$. Doxycycline inhibited the migration of A549 cells $(P<0.05)$. E. MMP-2 and MMP-9 were downregulated when either cell line was treated with doxycycline. Results are expressed as percentage of control. Similar results were obtained from three independent experiments, each performed in triplicate. Results show the means of the three experiments, and the error bars represent standard deviation $(* P<0.05$ and $* * P<0.01)$. 

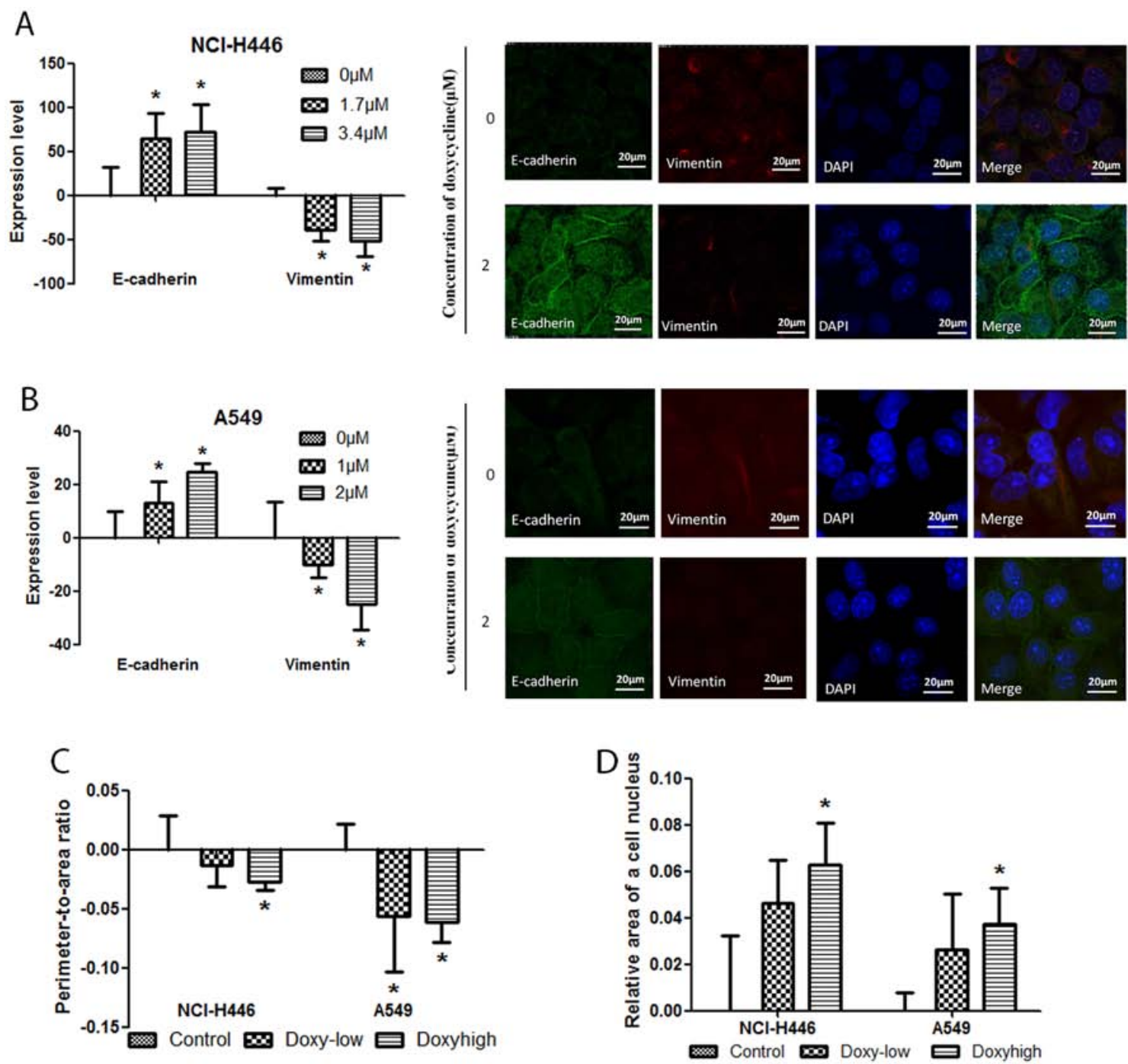

E

$\mathrm{F}$
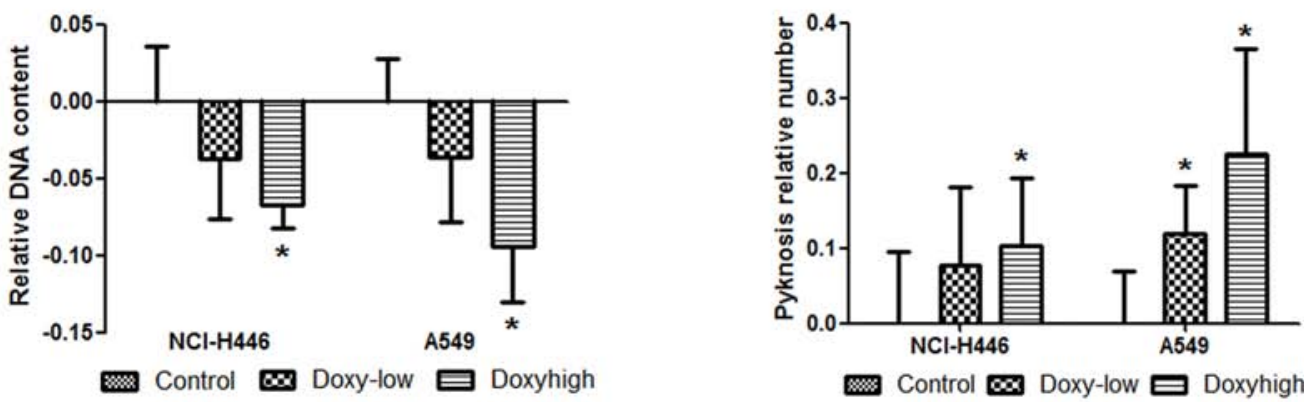

Figure 3: Effect of doxycycline on NCI-H446 and A549 cells visualized with high-content screening (HCS) systems and a fluorescence microscope. HCS detection of E-cadherin and vimentin was performed on NCI-H446 and A549 cells treated with $1.7 \mu \mathrm{M}$ or $3.4 \mu \mathrm{M}$ and $1 \mu \mathrm{M}$ or $2 \mu \mathrm{M}$ doxycycline (respectively) for $24 \mathrm{~h}$. Immunofluorescent staining was done for E-cadherin and vimentin of NCI-H446 and A549 cells treated with 0 or $2 \mu \mathrm{M}$ doxycycline for $24 \mathrm{~h}$. A. In NCI-H446 cells, and B. in A549 cells, E-cadherin levels were increased while vimentin levels were decreased with doxycycline treatment. C. The ratio of perimeter-to-area was decreased both in NCI-H446 and A549 cells treated with doxycycline. D. The relative area of nucleus was increased both in NCI-H446 and A549 cells treated with doxycycline. E. The DNA content in a cell was decreased both in NCI-H446 and A549 cells treated with doxycycline. F. The number of pyknosis was increased after doxycycline treatment. Standard error bars represent three independent experiments each performed in triplicate $\left({ }^{*} P<0.05\right.$ compared to the untreated control). 
A

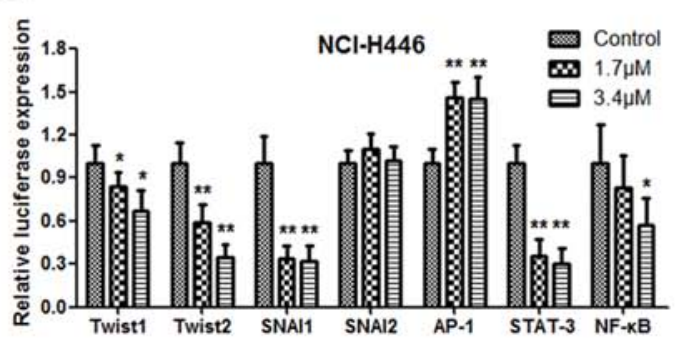

B

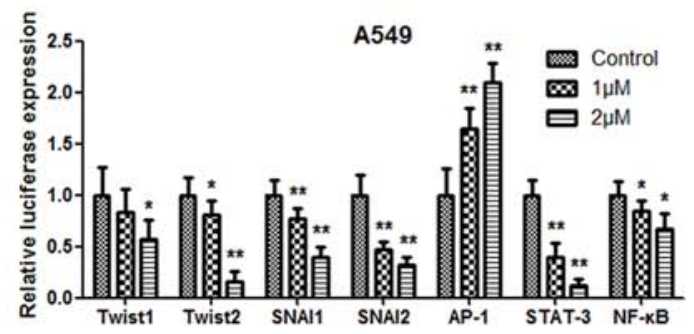

C

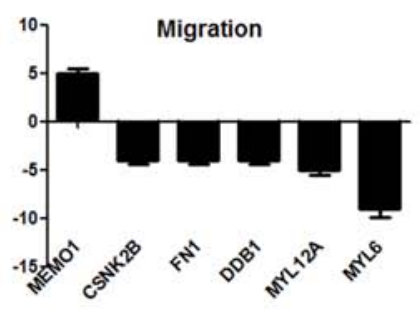

D

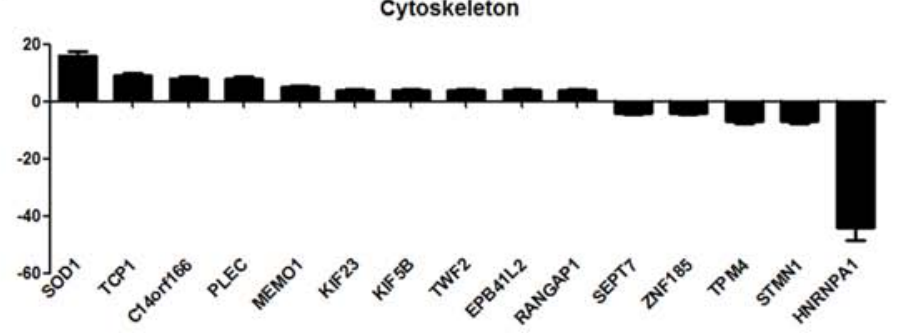

E

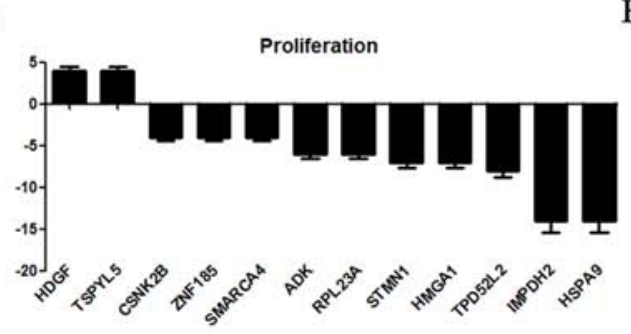

G

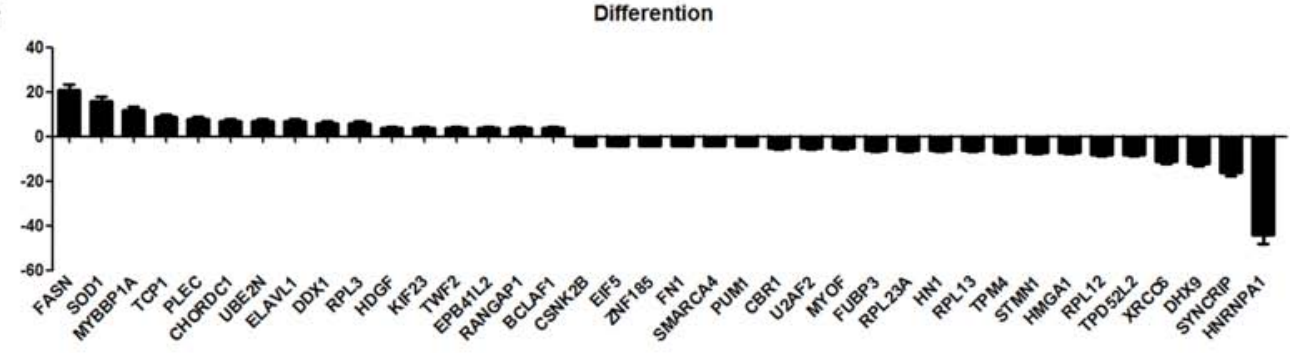

F

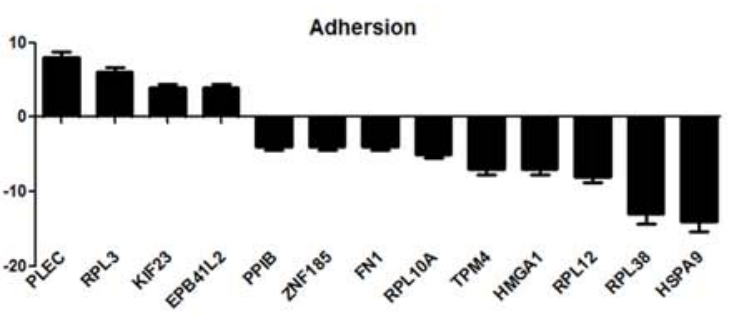

Differention

H

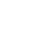




\section{Doxycycline has an antitumor effect in a mouse xenograft model}

We next examined the effects of doxycycline on spontaneous lung metastasis using NCI-H446 human lung cancer and Lewis lung carcinoma (LLC) xenografts in nude mice. Doxycycline treatment inhibited tumor growth in a dose-dependent manner. In particular, the NCI-H446 xenografts in the cyclophosphamide group died out before the end of the experiment (Fig. 5A \& 5C, 5E \& 5F). Body weight increased in the doxycycline-treated group (Fig. 5B $\& 5 \mathrm{D})$. The median survival time of the control groups of LLC xenografts was 15.5 days. When compared with the control group, the median survival times of high-dose, middle-dose, and low-dose doxycycline treatment groups were increased by $235 \%, 197 \%$, and $184 \%$, respectively, and the median survival time of the cyclophosphamide treatment group was increased by $171 \%$ (Fig. 5G). The number of tumors that shifted stoves was decreased from $22.4 \pm 7.96$ to $4.20 \pm 1.79$ in the lungs of nude mice with LLC xenografts treated with doxycycline, (Fig. 5H). These results strongly suggest that doxycycline inhibits tumor growth and metastasis.

\section{Doxycycline alters the expression of EMT markers in cancer tissues}

Immunohistochemical staining for E-cadherin, vimentin, MMP-2, and MMP-9 showed their expression to be correlated with doxycycline treatment. E-cadherin exhibited low expression, whereas vimentin, MMP-2, and MMP-9 exhibited high expression in tumor cells treated with doxycycline compared with the untreated group in both the membrane and cytoplasm (Fig. 6A \& 6B).

\section{DISCUSSION}

By preventing and suppressing tumor invasion and metastasis it may be possible to decrease the mortality rates of patients with malignant tumors [13]. Despite efforts to develop alternative treatments for lung cancer $[14,15]$, in many cases the cancers are refractory and there is still no effective therapy $[16,17,18,19]$.

Doxycycline has been shown to have high antimetastatic activity and low cytotoxicity in melanoma and breast carcinoma [20, 21]. In clinical trials with patients with pleural effusion, satisfactory results were achieved when doxycycline was used and there was inhibition of the activity of the suspended tumor cells. The current study focuses on the mechanism and anti-metastatic effects of doxycycline. We used several human cancer cell lines to investigate the effect of doxycycline on cell viability and the cell cycle. Our results showed that doxycycline inhibited the proliferation of NCI-H446 and A549 cells more than the other cell lines. Doxycycline has a strong inhibitory effect on malignancies originating from mesenchyme and neuroectoderm, including melanomas, endothelial cell tumors, and sarcomas [20, 22, 23]. All three kinds of lung cancer cells used in this study have mesodermal characteristics and readily undergo EMT [24-26]. NCI-H446 is a small cell lung cancer cell originating from neuroectoderm, while A549 cells are thought to have already undergone EMT. By contrast, lung squamous cell carcinoma is not very sensitive to doxycycline (unpublished data).

Doxycycline has a strong inhibitory effect on cells with mesenchymal characters. Early studies showed that doxycycline influences focal adhesion kinase (FAK) and cell adhesion and causes cells to lose anchors during migration [20]. Our current study does not examine whether doxycycline directly inhibits chemotaxis or indirectly inhibits chemotaxis by the inhibition of cell adhesion. It is possible that increased invasion and migration might due to increased generation of chemokines from tumor cells. Doxycycline also has other targets, including MMPs and E-cadherin, ultimately inhibiting metabolism and metastasis [27]. Two members of the MMP family, MMP-2 and MMP-9, are necessary for tumor invasion and metastasis [28, 29] [30]. Therefore, decreasing MMP activity could inhibit cancer cell invasion and metastasis [31,32]. We examined the regulation of MMP activity after doxycycline treatment. Our data show that doxycycline inhibits MMP-2 and MMP-9 activation, which is consistent with its inhibitory effects on metastasis.

EMT initiates tumor metastasis and alters cellcell adhesion [33-35]. E-cadherin and vimentin, two biomarkers of EMT, mediate cell adhesion. Downregulation of E-cadherin expression is both necessary and sufficient to confer metastatic ability to lung cancer cells that are otherwise non-metastatic $[36,37]$. Vimentin is an intermediate filament protein that, along with microtubules and actin filaments, forms the cytoskeleton. In some carcinomas, including prostatic carcinoma, vimentin is overexpressed and is therefore a marker for invasiveness and metastasis [38]. In this study, we found that doxycycline increased E-cadherin levels and decreased vimentin protein expression. This finding suggests that doxycycline suppresses EMT in lung cancer. In addition, studies of promoter activation and proteomics after doxycycline treatment revealed that doxycycline inhibits EMT-related transcription factor activity and that doxycycline exerts its antitumor effect by interfering with tumor cell EMT. These findings show that doxycycline acts upstream of EMT-related signal transduction to inhibit a wide range of cellular functions. The inhibitory effects of doxycycline on bacteria is derived from its interference with rhibosomes, which result in the inhibition of protein synthesis. After doxycycline treatment in cancer cell lines, RPLs were directly or indirectly regulated. Some RPLs were inhibited by doxycycline, such as RPL23A, RPL10A, RPL12, RPL38 and RPL13. On the contrary, 
A

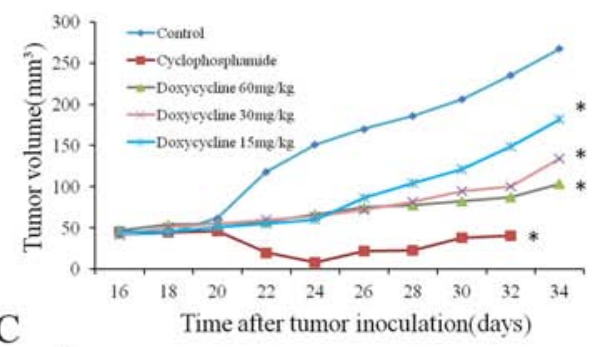

C

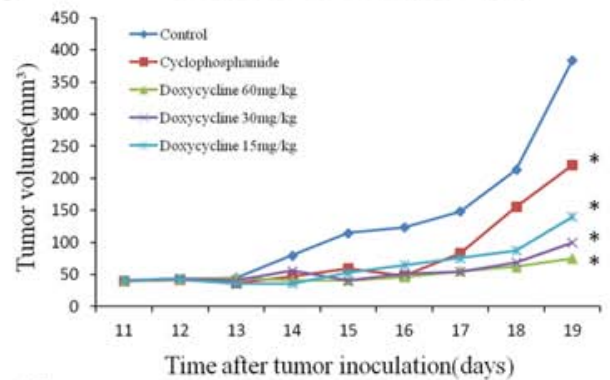

E

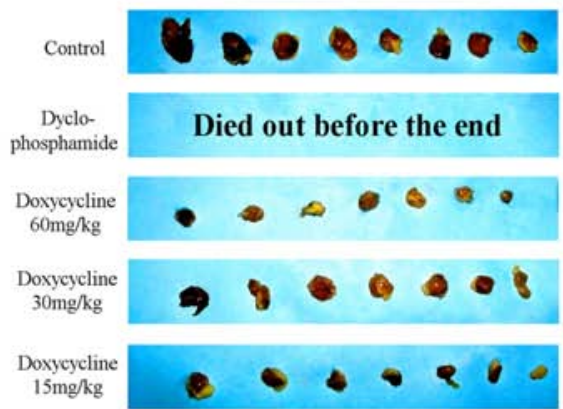

G

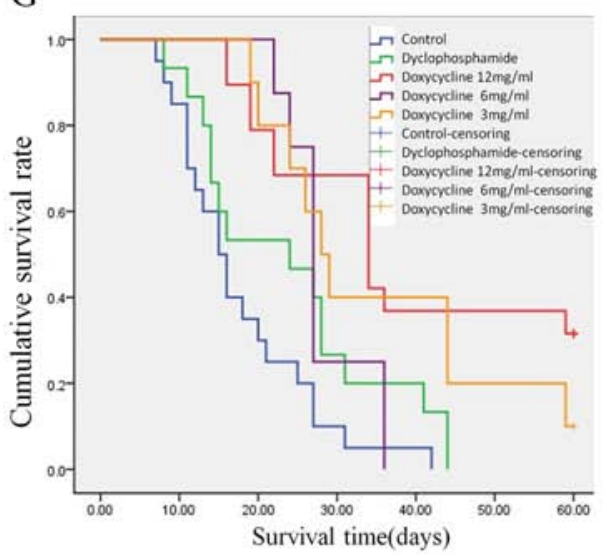

B

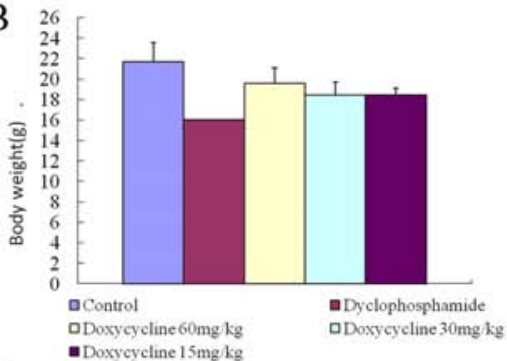

D

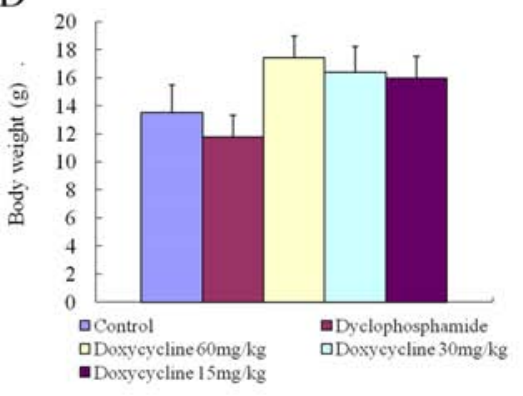

F

Control

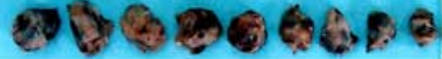

Dyclo-

phosphamide
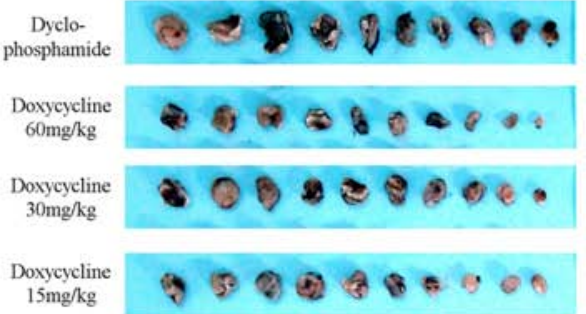

H
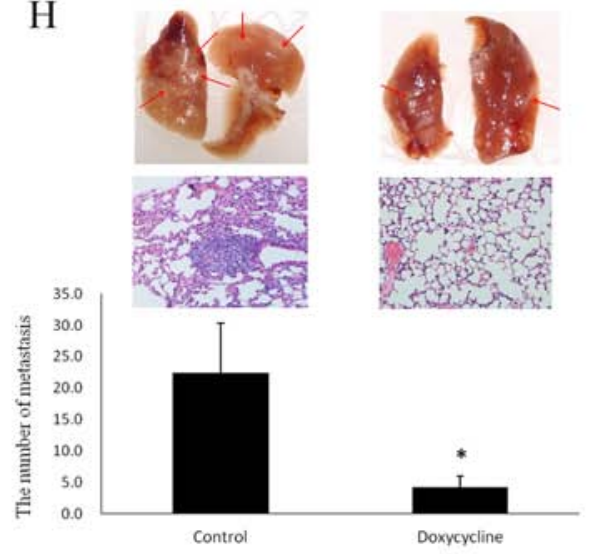

Figure 5: Effect of doxycycline on a nude mouse xenograft model. Mice were treated with saline, cyclophosphamide $(20 \mathrm{mg} / \mathrm{kg})$, or doxycycline $(60,30$, or $15 \mathrm{mg} / \mathrm{kg})$ for 5 weeks. A. Changes in tumor volume of NCI-H446 xenografts. Both doxycycline and cyclophosphamide diminished tumor volume of the tumors. B. Body weights (g) of animals with NCI-H446 xenografts. Doxycycline increased the body weights of nude mice with xenografts compared with cyclophosphamide. C. Changes in tumor volume of Lewis lung carcinoma (LLC) xenografts. Both doxycycline and cyclophosphamide diminished tumor volume. D. Body weights (g) of animals with LLC xenografts. Doxycycline increased the body weights of nude mice of xenografts compared with cyclophosphamide. E. Doxycycline treatment inhibited NCI-H446 xenografts growth in a dose-dependent manner. Particularly, the cyclophosphamide xenografts died out before the end of the experiment because of toxicity. F. Doxycycline treatment inhibited LLC xenografts growth in a dose-dependent manner. G. The median survival time of control groups of LLC xenografts is 15.5 days. The median survival times of high-dose, middle-dose, and low-dose doxycycline groups were $235 \%, 197 \%$, and $184 \%$, respectively, compared with the control group. By contrast, the median survival time of the cyclophosphamide treatment was increased by $171 \%$. $\mathbf{H}$. The number of tumors that shifted stoves markedly decreased in lungs of nude mice with LLC xenografts. Each experiment was performed in triplicate. Results show the means of the three experiments, and the error bars represent standard deviation $(* P<0.05)$. 

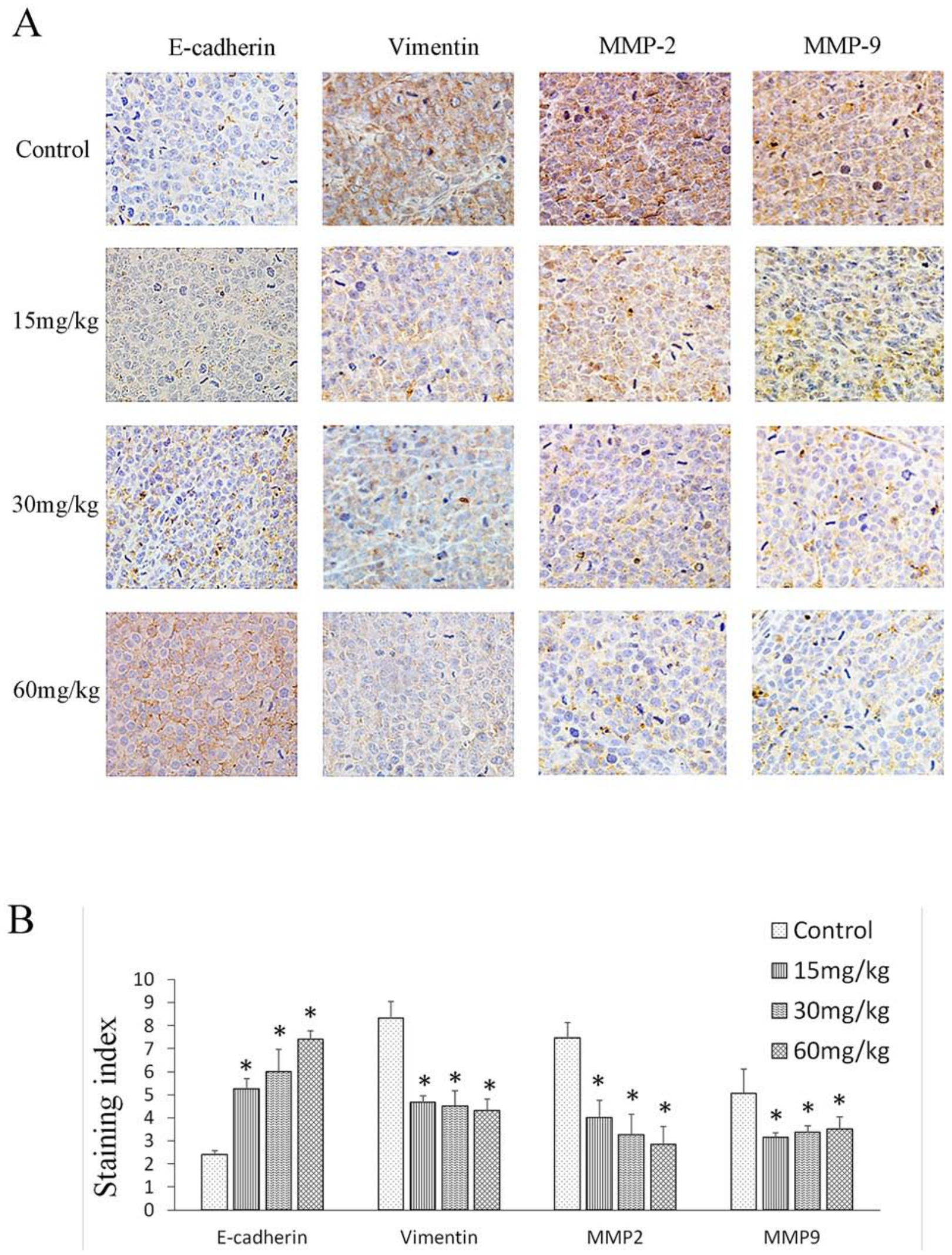

Figure 6: Effect of doxycycline on EMT protein expression. Brown or yellow staining was observed in the cytoplasm or nucleus. E-cadherin was located in membranes, and vimentin, MMP-2, and MMP-9 were found in the cytoplasm. A. Representative photographs of treated and untreated cells. B. Doxycycline-treated sections had weaker vimentin, MMP-2, and MMP-9 staining but stronger E-cadherin staining when compared with the sections obtained from control mice. Each experiment was performed in triplicate. Results show the means of the three experiments, and the error bars represent standard deviation $(* P<0.05)$ 
RPL3 was compensatorily increased to maintain basic protein synthesis. As shown in Table S1, many molecular and biological functions of these RPLs are similar even the same between Eukaryotes and prokaryotes. In summary, doxycycline may be an effective alternative treatment for persistent carcinoma, as well as a new candidate for adjuvant chemoradiotherapy.

\section{MATERIALS AND METHODS}

\section{Materials}

Crystal violet, doxycycline hydrochloride, and 3-(4,5-dimethylthiazol-2-y1)-2,5-diphenyltetrazolium bromide (MTT) were purchased from Sangon Biotech (Shanghai, China). Matrigel and transwell chambers were purchased from BD Biosciences (San Jose, CA, USA). Propidium iodide (PI) was purchased from Beyotime Biotech (Jiangsu, China). The antibodies to MMP-2, MMP-9, vimentin, and E-cadherin were purchased from Affinity Bioreagents (Colorado, USA). Rabbit polyclonal anti-E-cadherin and rabbit polyclonal anti-MMP-9 were purchased from ZSGB-BIO (Beijing, China). Fluorescein isothiocyanate AffiniPure Goat Anti-Rabbit $\operatorname{IgG}(\mathrm{H}+\mathrm{L})$ secondary antibodies were purchased from EarthOx (San Francisco, USA). Dual luminescence assay kit was purchased from GeneCopoeia (Guangzhou, China).

\section{Cell culture}

The following human cell lines were obtained KeyGen Biotech (Nanjing, China): NCI-H446, and A549 lung cancer cell lines; PLC, SMMC7721, HepG2, $\mathrm{MHCC} 97 \mathrm{H}$, and MHCC97L hepatoma carcinoma lines; LOVO colon cancer line; PC-3 prostate cancer cell line; A875, A375, Mum2B, and Mum2C melanoma lines; MCF-7 and MDA-MB-231 breast cancer cell lines; SGC-7901 gastric cancer cell line; PCNA-1 and AsPC-1 pancreatic cancer cell lines; HeLa cervical cancer line; K562 and HL60 leukemia lines; and SH-SY5Y neuroblastoma line. Cells were cultured in medium supplemented with $10 \%$ heat-inactivated $\left(56^{\circ} \mathrm{C}, 30 \mathrm{~min}\right)$ fetal calf serum (Hyclone, USA) and maintained at $37^{\circ} \mathrm{C}$ with $5 \% \mathrm{CO}_{2}$ in a humidified atmosphere. Details are shown in Table S2.

\section{Cell viability assay}

Cell viability was determined by MTT assay. Cells $\left(5 \times 10^{3}\right.$ cells $\left./ \mathrm{mL}\right)$ were seeded in 96 -well culture plates. After overnight incubation, cells were treated with various concentrations of doxycycline hydrochloride. After $48 \mathrm{~h}$ incubation, cell viability was measured after the addition of $20 \mu \mathrm{L}$ MTT at $37^{\circ} \mathrm{C}$ for $4 \mathrm{~h}$. Afterward, $150 \mu \mathrm{L}$ dimethyl sulfoxide was added to dissolve the formazan crystals. Optical density was measured at $570 \mathrm{~nm}$ with a microplate reader (Multiskan ${ }^{\mathrm{TM}} \mathrm{FC}$, Thermo Scientific, Waltham, MA, USA).

\section{Wound-healing assay}

NCI-H446 and A549 cells were grown on a $35 \mathrm{~mm}$ dish to $100 \%$ confluence and then scratched to form a $100-\mu \mathrm{m}$ wound using sterile pipette tips. The cells were then cultured in the presence or absence of doxycycline hydrochloride in serum-free media for $24 \mathrm{~h}$. Images of the cells were taken at 24 and $48 \mathrm{~h}$ using a light microscope (Nikon, Japan).

\section{Invasion assays}

Cell invasion assays were performed using a transwell chamber inserted with a polyethylene terephthalate filter membrane containing $8.0 \mu \mathrm{m}$ pores in 24-well plates (Corning, USA). For cell invasion assays, the filter membranes were coated with Matrigel. Cells $\left(1 \times 10^{5}\right.$ cells $\left./ \mathrm{mL}\right)$ suspended in $200 \mu \mathrm{L}$ of serumfree medium were seeded onto the upper compartment of the transwell chamber. The lower chamber was filled with medium containing $10 \%$ fetal bovine serum (as chemoattractant for migrated and invaded cancer cells) and various concentrations of doxycycline hydrochloride. After incubation for $24 \mathrm{~h}$, the medium in the upper chamber was removed, and the filters were fixed with $10 \%$ methanol for $20 \mathrm{~min}$. The cells remaining on the upper surface of the filter membrane were then completely removed by wiping with a cotton swab, and the cells on the opposite surface of the filter membrane were stained with $0.1 \%$ crystal violet for $10 \mathrm{~min}$. The invading cells were then visualized and counted from six randomly selected fields using an inverted microscope at $100 \times$ magnification.

\section{Fluorescence-activated cell sorting (FACS) analysis}

NCI-H446 and A549 cells were harvested separately and washed twice with phosphate buffered saline (PBS). The cells were then fixed with cold $70 \%$ ethanol for $12 \mathrm{~h}$ at $4{ }^{\circ} \mathrm{C}$. Afterward, the cells were washed with $1 \mathrm{~mL}$ PBS. The cells were treated with $500 \mu \mathrm{L}$ PBS containing $100 \mu \mathrm{g} / \mathrm{mL}$ ribonuclease and $50 \mu \mathrm{g} / \mathrm{mL}$ PI. The cells were analyzed by flow cytometry (Millipore guava easyCyte ${ }^{\mathrm{TM}}$ ).

\section{Immunofluorescent staining}

NCI-H446 and A549 cells $\left(4 \times 10^{3}\right.$ cells $\left./ \mathrm{mL}\right)$ were seeded in 96-well culture plates. After overnight incubation, the cells were treated with various concentrations of doxycycline hydrochloride. After incubation for $24 \mathrm{~h}$, cells were washed twice in PBS, fixed with $10 \%$ formalin in PBS, permeabilized, and blocked with PBS containing NP-40 (0.1\%) and bovine serum albumin (3\%). The cells were then incubated in the same 
solution containing primary antibodies specific for either E-cadherin antibody (1:50 dilution) or vimentin antibody (1:50 dilution) for $1 \mathrm{~h}$ at room temperature $\left(25^{\circ} \mathrm{C}\right)$. Cells were washed four times in PBS and incubated in secondary antibody (1:200 dilution) for $30 \mathrm{~min}$ at room temperature. Cells were then washed four times in PBS and covered with Hoechst 33342 dye for $30 \mathrm{~min}$ at room temperature. Cells were washed four additional times in PBS then proteins were visualized with high-content screening (HCS) systems.

\section{Gelatin zymography assay}

NCI-H446 and A549 cells $\left(1 \times 10^{6}\right.$ cells/well $)$ were plated in 12-well plates and incubated in serumfree RPMI 1640 medium in the presence of doxycycline hydrochloride for $24 \mathrm{~h}$. At the end of incubation, the conditioned medium was harvested, placed on $10 \%$ sodium dodecyl sulfate (SDS)-polyacrylamide gel containing $0.2 \%$ gelatin (Sigma-Aldrich Corp), and then separated by electrophoresis. The gels were soaked in $2.5 \%$ Triton $\mathrm{X}-100$ in deionized water twice for $60 \mathrm{~min}$ at $25^{\circ} \mathrm{C}$ to remove SDS. Gels were incubated at $37^{\circ} \mathrm{C}$ with substrate buffer (50 mM Tris $\mathrm{HCl}, 5 \mathrm{mM} \mathrm{CaCl}_{2}, 0.02 \% \mathrm{NaN}_{3}$, and $1 \%$ Triton $\mathrm{X}-100, \mathrm{pH} 8.0$ ) for $18 \mathrm{~h}$. The gel was stained using $0.2 \%$ Coomassie blue for $1 \mathrm{~h}$ and destained in water containing $10 \%$ acetic acid and 50\% methanol. Bands corresponding to the activity of MMP-2 and MMP-9 were quantified with ImageJ (National Institutes of Health).

\section{Dual-luciferase assay}

NCI-H446 and A549 cells were transfected with dual-reporter constructs using transfection reagents. After changing to fresh medium $24 \mathrm{~h}$ after transfection, cells were treated with various concentrations of doxycycline hydrochloride. After $48 \mathrm{~h}$, the culture medium was collected into a 96-well white plate and luminescence measured with a luminometer. Details are shown in Table S3 and S4.

\section{Multidimensional liquid chromatography- tandem mass spectrometry}

NCI-H446 and A549 cells $\left(4 \times 10^{3}\right.$ cells $\left./ \mathrm{mL}\right)$ were seeded in a $100 \mathrm{~mm}$ dish to $70-80 \%$ confluence. The cells were then cultured in the presence $(3.4 \mu \mathrm{M})$ or absence of doxycycline hydrochloride for $24 \mathrm{~h}$. After cell lysis, samples were tested with multidimensional liquid chromatography-tandem mass spectrometry.

\section{Animal studies}

Male BAlB/c nu/nu mice, 5-6 weeks old, were maintained in a specific pathogen-free animal care facility according to institutional guidelines. Xenografts of tumors were established by subcutaneous injection of $1 \times 10^{7}$ cells (suspended in PBS) into the flank. One day after tumor cell inoculation, the mice were randomly divided into 5 groups ( $n=10$ /group). After the tumors reached an approximate volume of $100 \mathrm{~mm}^{3}$ (approximately six weeks after injection), the mice were treated with 60,30 , or $15 \mathrm{mg} / \mathrm{kg}$ of doxycycline; $20 \mathrm{mg} / \mathrm{kg}$ of cyclophosphamide; or saline by oral gavage once a day. Body weights were measured at different time points after tumor cell inoculation. Tumor diameters were measured every day, and tumor volumes were calculated according to the formula $V=\mathrm{ab}^{2} / 2$ ( $a=$ length of tumor, $b=$ width of tumor). Seven weeks after treatment, all mice were euthanized and both xenografts and lungs were resected and measured. Lung tissue was harvested for histologic examination and the nodes in lungs were observed using a stereoscopic microscope. Metastases from xenograft to lungs were measured after HE staining.

Another 50 mice were allocated randomly to 5 groups as described above (10 mice per group), in order to measure survival rates. Each mouse was injected $1 \times 10^{7}$ cells (suspended in PBS) in the caudal vein. The survival time of every mouse were recorded.

\section{Immunohistochemical analysis}

Fresh tissues from mice were fixed in $4 \%$ paraformaldehyde, embedded in paraffin, cut into $4 \mu \mathrm{m}$ thick slices, and placed on slides. The tissues were deparaffinized with xylene, dehydrated in decreasing concentrations of ethanol, and subsequently incubated with $3 \%$ hydrogen peroxide for 15 min to block endogenous peroxidase activity. For antigen retrieval, tissues were treated with citrate buffered saline $(\mathrm{pH} 6.0)$ for $15 \mathrm{~min}$ at $95^{\circ} \mathrm{C}$. Tissues were incubated with normal goat serum for $20 \mathrm{~min}$ at room temperature to block unspecific labeling and then incubated with the following primary antibodies in a humidified chamber overnight at $4^{\circ} \mathrm{C}$ : rabbit polyclonal anti-E-cadherin (Zhongshan, ready-to-use), goat polyclonal anti-vimentin (Affinity, dilution 1:50), and rabbit polyclonal anti-MMP-9 (Zhongshan, ready-to-use). Diaminobenzidine and hematoxylin were used for color development and as counterstain, respectively. Expression of E-cadherin and vimentin were independently evaluated by two investigators. Tumor cells with brown staining of the cytoplasm, nucleus or membrane were considered positive and then scored based on four classes: none (0), weak brown $(1+)$, moderate brown $(2+)$, and strong brown $(3+)$. The percentage of stained tumor cells was divided into five classes: 0 for negative cells, 1 for $1-25 \%, 2$ for $25-50 \%, 3$ for $50-75 \%$, and 4 for $>75 \%$.

\section{Statistical analyses}

All data are expressed as means \pm standard deviation. Comparisons between groups were performed by one-way analysis of variance followed by Bonferroni 
post hoc test (SPSS software package version 17.0, SPSS Inc., Chicago, IL, USA). The level of significance was set at $P<0.05$.

\section{ACKNOWLEDGMENTS}

This study was supported by the National Natural Science Funds of China [Grants 81201650 and 81572838]; the Key Technologies R\&D Program of Tianjin [Grant 11ZCKFSY06900]; Tianjin Natural Science and Technology Fund [Grant 15JCYBJC26400]; and Foundation for the Author of National Excellent Doctoral Dissertation of China [Grant 201482]; and National Science and Technology Major Project of China [Grant 2013ZX09301306]

\section{CONFLICTS OF INTEREST} interests.

The authors declare that they have no competing

\section{REFERENCES}

1. Lamb R, Ozsvari B, Lisanti CL, Tanowitz HB, Howell A, Martinez-Outschoorn UE, Sotgia F and Lisanti MP. Antibiotics that target mitochondria effectively eradicate cancer stem cells, across multiple tumor types: treating cancer like an infectious disease. Oncotarget. 2015; 6:4569-4584.

2. Richards C, Pantanowitz L and Dezube BJ. Antimicrobial and non-antimicrobial tetracyclines in human cancer trials. Pharmacological research: the official journal of the Italian Pharmacological Society. 2011; 63:151-156.

3. Lokeshwar BL. Chemically modified non-antimicrobial tetracyclines are multifunctional drugs against advanced cancers. Pharmacological research: the official journal of the Italian Pharmacological Society. 2011; 63:146-150.

4. Pulvino M, Chen L, Oleksyn D, Li J, Compitello G, Rossi R, Spence S, Balakrishnan V, Jordan C, Poligone B, Casulo C, Burack R, Shapiro JL, Bernstein S, Friedberg JW, Deshaies RJ, et al. Inhibition of COP9-signalosome (CSN) deneddylating activity and tumor growth of diffuse large B-cell lymphomas by doxycycline. Oncotarget. 2015; 6:14796-14813.

5. Saikali $Z$ and Singh G. Doxycycline and other tetracyclines in the treatment of bone metastasis. Anti-cancer drugs. 2003; 14:773-778.

6. Sun B, Zhang S, Zhang D, Yin X, Wang S, Gu Y and Wang Y. Doxycycline influences microcirculation patterns in B16 melanoma. Experimental biology and medicine. 2007; 232:1300-1307.

7. Wu W, Yu LH, Ma B and Xu MJ. The inhibitory effect of doxycycline on cisplatin-sensitive and -resistant epithelial ovarian cancer. PloS one. 2014; 9:e89841.
8. Lamb R, Fiorillo M, Chadwick A, Ozsvari B, Reeves KJ, Smith DL, Clarke RB, Howell SJ, Cappello AR, MartinezOutschoorn UE, Peiris-Pages M, Sotgia F and Lisanti MP. Doxycycline down-regulates DNA-PK and radiosensitizes tumor initiating cells: Implications for more effective radiation therapy. Oncotarget. 2015; 6:14005-14025.

9. Khan $\mathrm{N}$ and Mukhtar H. Dietary agents for prevention and treatment of lung cancer. Cancer letters. 2015; 359:155-164.

10. Becker $\mathrm{K}$ and $\mathrm{Xu} \mathrm{Y}$. Management of tyrosine kinase inhibitor resistance in lung cancer with EGFR mutation. World journal of clinical oncology. 2014; 5:560-567.

11. Wan J, Che Y, Kang N and Wu W. SOCS3 blocks HIF1alpha expression to inhibit proliferation and angiogenesis of human small cell lung cancer by downregulating activation of Akt, but not STAT3. Molecular medicine reports. 2015; 12:83-92.

12. Hamilton $\mathrm{G}$, Burghuber $\mathrm{O}$ and Zeillinger R. Circulating tumor cells in small cell lung cancer: ex vivo expansion. Lung. 2015; 193:451-452.

13. $\mathrm{Wu} \mathrm{W}$, Bi C, Credille KM, Manro JR, Peek VL, Donoho GP, Yan L, Wijsman JA, Yan SB and Walgren RA. Inhibition of tumor growth and metastasis in non-small cell lung cancer by LY2801653, an inhibitor of several oncokinases, including MET. Clinical cancer research: an official journal of the American Association for Cancer Research. 2013; 19:5699-5710.

14. Zhou QM, Zhang H, Lu YY, Wang XF and Su SB. Curcumin reduced the side effects of mitomycin $\mathrm{C}$ by inhibiting GRP58-mediated DNA cross-linking in MCF-7 breast cancer xenografts. Cancer science. 2009; 100:2040-2045.

15. Zhou QM, Wang XF, Liu XJ, Zhang H, Lu YY and Su SB. Curcumin enhanced antiproliferative effect of mitomycin $\mathrm{C}$ in human breast cancer MCF-7 cells in vitro and in vivo. Acta pharmacologica Sinica. 2011; 32:1402-1410.

16. Wheler JJ, Tsimberidou AM, Hong DS, Naing A, Falchook GS, Fu S, Moulder S, Stephen B, Wen S and Kurzrock R. Risk of serious toxicity in 1181 patients treated in phase I clinical trials of predominantly targeted anticancer drugs: the M. D. Anderson Cancer Center experience. Annals of oncology : official journal of the European Society for Medical Oncology / ESMO. 2012; 23:1963-1967.

17. Morabito A, Carillio G, Daniele G, Piccirillo MC, Montanino A, Costanzo R, Sandomenico C, Giordano P, Normanno N, Perrone F, Rocco G and Di Maio M. Treatment of small cell lung cancer. Critical reviews in oncology/hematology. 2014; 91:257-270.

18. Duffy MJ, McGowan PM and Gallagher WM. Cancer invasion and metastasis: changing views. The Journal of pathology. 2008; 214:283-293.

19. Sun T, Sun BC, Ni CS, Zhao XL, Wang XH, Qie S, Zhang DF, Gu Q, Qi H and Zhao N. Pilot study on the interaction between B16 melanoma cell-line and bone-marrow 
derived mesenchymal stem cells. Cancer letters. 2008; 263:35-43.

20. Sun T, Zhao N, Ni CS, Zhao XL, Zhang WZ, Su X, Zhang DF, Gu Q and Sun BC. Doxycycline inhibits the adhesion and migration of melanoma cells by inhibiting the expression and phosphorylation of focal adhesion kinase (FAK). Cancer letters. 2009; 285:141-150.

21. Liu S, Liu X, Wang H, Zhou Q, Liang Y, Sui A, Yao R, Zhao B and Sun M. Lentiviral vector-mediated doxycycline-inducible USP39 shRNA or cDNA expression in triple-negative breast cancer cells. Oncology reports. 2015; 33:2477-2483.

22. Fainaru O, Adini I, Benny O, Bazinet L, Pravda E, D'Amato R and Folkman J. Doxycycline induces membrane expression of VE-cadherin on endothelial cells and prevents vascular hyperpermeability. FASEB journal: official publication of the Federation of American Societies for Experimental Biology. 2008; 22:3728-3735.

23. Roomi MW, Kalinovsky T, Rath M and Niedzwiecki A. In vitro modulation of MMP-2 and MMP-9 in pediatric human sarcoma cell lines by cytokines, inducers and inhibitors. International journal of oncology. 2014; 44:27-34.

24. Ko H. Geraniin inhibits TGF-beta1-induced epithelialmesenchymal transition and suppresses A549 lung cancer migration, invasion and anoikis resistance. Bioorganic \& medicinal chemistry letters. 2015; 25:3529-3534.

25. Wu TH, Chou YW, Chiu PH, Tang MJ, Hu CW and Yeh ML. Validation of the effects of TGF-beta1 on tumor recurrence and prognosis through tumor retrieval and cell mechanical properties. Cancer cell international. 2014; 14:20.

26. Cao J, Song Y, Bi N, Shen J, Liu W, Fan J, Sun G, Tong T, He J, Shi Y, Zhang X, Lu N, He Y, Zhang H, Ma K, Luo X, et al. DNA methylation-mediated repression of miR-886-3p predicts poor outcome of human small cell lung cancer. Cancer research. 2013; 73:3326-3335.

27. Chen Z, Wang Y, Liu W, Zhao G, Lee S, Balogh A, Zou Y, Guo Y, Zhang Z, Gu W, Li C, Tigyi G and Yue J. Doxycycline inducible Kruppel-like factor 4 lentiviral vector mediates mesenchymal to epithelial transition in ovarian cancer cells. PloS one. 2014; 9:e105331.

28. Shay G, Lynch CC and Fingleton B. Moving targets: Emerging roles for MMPs in cancer progression and metastasis. Matrix biology : journal of the International Society for Matrix Biology. 2015; 44-46C:200-206.

29. Rowe RG and Weiss SJ. Navigating ECM barriers at the invasive front: the cancer cell-stroma interface.
Annual review of cell and developmental biology. 2009; 25:567-595.

30. Sani IK, Marashi SH and Kalalinia F. Solamargine inhibits migration and invasion of human hepatocellular carcinoma cells through down-regulation of matrix metalloproteinases 2 and 9 expression and activity. Toxicology in vitro: an international journal published in association with BIBRA. 2015; 29:893-900.

31. Ma CY, Ji WT, Chueh FS, Yang JS, Chen PY, Yu CC and Chung JG. Butein inhibits the migration and invasion of SK-HEP-1 human hepatocarcinoma cells through suppressing the ERK, JNK, p38, and uPA signaling multiple pathways. Journal of agricultural and food chemistry. 2011; 59:9032-9038.

32. Liao CL, Lai KC, Huang AC, Yang JS, Lin JJ, Wu SH, Gibson Wood W, Lin JG and Chung JG. Gallic acid inhibits migration and invasion in human osteosarcoma U-2 OS cells through suppressing the matrix metalloproteinase-2/-9, protein kinase $\mathrm{B}$ (PKB) and PKC signaling pathways. Food and chemical toxicology : an international journal published for the British Industrial Biological Research Association. 2012; 50:1734-1740.

33. Tiwari N, Gheldof A, Tatari M and Christofori G. EMT as the ultimate survival mechanism of cancer cells. Seminars in cancer biology. 2012; 22:194-207.

34. Kumar S, Das A and Sen S. Extracellular matrix density promotes EMT by weakening cell-cell adhesions. Molecular bioSystems. 2014; 10:838-850.

35. Sun T, Sun BC, Zhao XL, Zhao N, Dong XY, Che N, Yao Z, Ma YM, Gu Q, Zong WK and Liu ZY. Promotion of tumor cell metastasis and vasculogenic mimicry by way of transcription coactivation by Bcl-2 and Twist1: a study of hepatocellular carcinoma. Hepatology. 2011; 54:1690-1706.

36. Baranwal S and Alahari SK. Molecular mechanisms controlling E-cadherin expression in breast cancer. Biochemical and biophysical research communications. 2009; 384:6-11.

37. Sun T, Zhao N, Zhao XL, Gu Q, Zhang SW, Che N, Wang XH, Du J, Liu YX and Sun BC. Expression and functional significance of Twist1 in hepatocellular carcinoma: its role in vasculogenic mimicry. Hepatology. 2010; 51:545-556.

38. Zhao Y, Yan Q, Long X, Chen X and Wang Y. Vimentin affects the mobility and invasiveness of prostate cancer cells. Cell biochemistry and function. 2008; 26:571-577. 